\title{
On the human control of vehicles: an experimental study of acceleration
}

\author{
Paolo Bosetti • Mauro Da Lio • Andrea Saroldi
}

Received: 7 June 2013 / Accepted: 17 September 2013 / Published online: 28 September 2013

(C) The Author(s) 2013. This article is published with open access at SpringerLink.com

\begin{abstract}
This paper presents an experimental investigation of human control of vehicles carried out on the basis of general theories on human movement. The longitudinal and lateral accelerations are studied, and their relations with theories of motor optimality principles, such as minimum jerk, minimum variance, and the two-thirds power law are highlighted. Data have been collected during the final experimental phase of the EU interactIVe project, in which a vehicle developed by Centro Ricerche Fiat has been used to demonstrate driver continuous support produced by an artificial codriver, within a shared initiative framework. 24 subjects drove the vehicle on a test route twice: once with the system active, the other with the system silent. The test route is composed of urban arterials, extra urban and motorway roads, and takes approximately $40-45 \mathrm{~min}$ to be driven. The total database thus amounts to $\sim 35 \mathrm{~h}$ of driving data recordings, for a total of $\sim 1.2 \mathrm{M}$ samples per signal. Statistical summary data are presented, which describe human preferred accelerations, correlation between acceleration, curvature, and speed, and between longitudinal and lateral acceleration. Different driving modalities, corresponding to different motor strategies and primitives, are revealed. Comparisons with literature data are also made and discussed. The summary statistics may be useful for the design of future ADAS systems, and indeed they have been collected for the final tuning of the interactIVe co-driver.
\end{abstract}

\footnotetext{
P. Bosetti $(\bowtie) \cdot$ M. Da Lio

Department of Industrial Engineering, University of Trento,

Trento, Italy

e-mail: paolo.bosetti@unitn.it

M. Da Lio

e-mail: mauro.dalio@unitn.it

A. Saroldi

Centro Ricerche Fiat, 10043 Orbassano, Italy

e-mail: andrea.saroldi@crf.it
}

Keywords Driver modeling $\cdot$ Intelligent vehicles $\cdot$ Human machine interaction - Advanced driver assistance systems . Man-machine systems

\section{Human sensory-motor strategies}

THE understanding of human movement plays a central role in many application domains. Recent theories say that the human brain motor system is active in several covert (non-executed) motor activities, such as motion planning and observation of other people movements (mirroring) [1-3]. It is believed that the ability to predict how a person would move - given an objective and in conjunction with the observation of other people actual movements - is at the origin of the understanding of intentions [4], empathy, and ultimately social interactions [5]. Such a framework has also been adopted for humanrobot interactions [5, 6]. Within the EU interactIVe project [7] the Authors adopted the same conceptual framework for developing an artificial cognitive system (named co-driver) able to understand the driver intentions and to produce a variety of Driver Assistance Functions [8-10].

Several authors showed that general human sensory-motor strategies are learnt and optimized [11-15]. Human movements - as for example the task of reaching an object are typically carried out as optimized motor units [16, 17], which are sent to execution in a feed-forward fashion, and while still in execution, they may be updated. Updating corrects only task-relevant deviations (i.e. the goal is pursued from the deviated position, without returning to the previous planned trajectory), which is known as minimum intervention principle [12].

The optimization criterion is most often said to be minimum jerk, and in facts human movements are smooth. However, further studies have shown that minimum jerk may be a byproduct of another optimality criterion, which is minimum 
variance $[15,18]$. According to this criterion, humans learn how to control movements so that the effect of motor neuron noise is minimized, and thus they achieve the best tradeoff between accuracy and speed.

Human movement is also known to withstand a velocitycurvature-acceleration constraint known as the two-thirds power law, which states that while hand-tracing an arc, the angular velocity is limited by the two thirds power of the local curvature $[19,20]$. As for the jerk, this is believed to be a byproduct of the same minimum variance principle $[15,18,21]$.

The problem of producing a body movement - such as getting a hand to a desired target - subject to the minimum variance principle is an optimal control problem. The simulation theory of cognition [1] says that humans learn forward and inverse models of the plants they are going to control $[22,23]$ (starting with, but not limited to, their own body), so that sensorial consequences of actions may be predicted, and actions that achieve desired perceptual goals may be produced $[1,3]$.

In this conceptual framework, the control of vehicles may be seen as a particular case of plant control (in control theory, "plant" means the dynamical system to be controlled), achieved by learning forward and inverse models of the vehicle dynamics. This justifies the current opinion in vehicle dynamics that drivers have mental models of the vehicles, used to anticipate the effect of control $[24,25]$. Optimality principles that lead to efficient control of the human body may be reasonably postulated for the control of vehicles too. It is thus no surprise that optimal control and model predictive control approaches have been successfully used to model drivers [24, 25], including those presented in Authors' previous works [8, 26-30].

Moreover, road bends are driven with a limiting lateral acceleration that decreases with curvature [28, 31-38], which is analogous of the two-thirds power law. Some authors explained the speed-curvature correlation as a way of minimizing the effects of steering errors $[32,36]$, which is the same conceptual argument of minimum variance criterion used to explain the origin of the two-thirds power law.

There is consequently a theoretical justification for looking at the curvature-acceleration-speed relationships as just another facet of more general human motor optimality criteria and put it in relation with the two-thirds power law.

As for what concerns the longitudinal control, the accelerations used in human driving may also be found in some previous studies of Adaptive Criuse Control (ACC) systems $[39,40]$, while correlations between longitudinal and lateral acceleration are pointed out in other papers [28, 41-43]. The data collected and presented in the present work will be compared to this literature references in the following sections.

As a final theoretical consideration, it is worth recalling the hierarchical nature of human behaviors, of which driving is one case. The recently proposed Extended Control Model (ECOM) $[44,45]$ explains the driving action as a combination of a number of concurrent processes, hierarchically organized.
High-level processes are long-term tasks that control lowlevel tasks - such as tactical maneuvering - which in turn control motor primitives that are simple perception-action units. The ECOM model may be seen as an evolution of precedent models such as the Michon model [46], framed within embodied cognition. Regardless of the number and nature of the model layers (the ECOM assumes 4 layers), the important point is that higher levels in the cognitive architecture switch on and off different motor primitives. One may thus observe different ways of driving (either motor units or entire behaviors) depending on ECOM states and goals at the various layers. An important example will be discussed hereafter.

\section{Experiment description}

\subsection{Test route}

The test route is depicted in Fig. 1 and is available interactively on a map online [47]. It is made of urban arterials, extraurban roads and motorways, with roundabouts, ramps, and intersections. The course starts at CRF headquarters $(a)$ and follows the letters in alphabetic order to $g$, then returns to $a$. The motorway section is $c-d$-e.

The total route length is $53 \mathrm{~km}$, which are typically driven in 40-45 min. The main track characteristics are listed Table 1.

\subsection{Test vehicle}

The test vehicle is one of the demonstrator vehicles developed within the interactIVe project [7], namely the CRF one. It is a Lancia Delta with a co-driver [8-10].

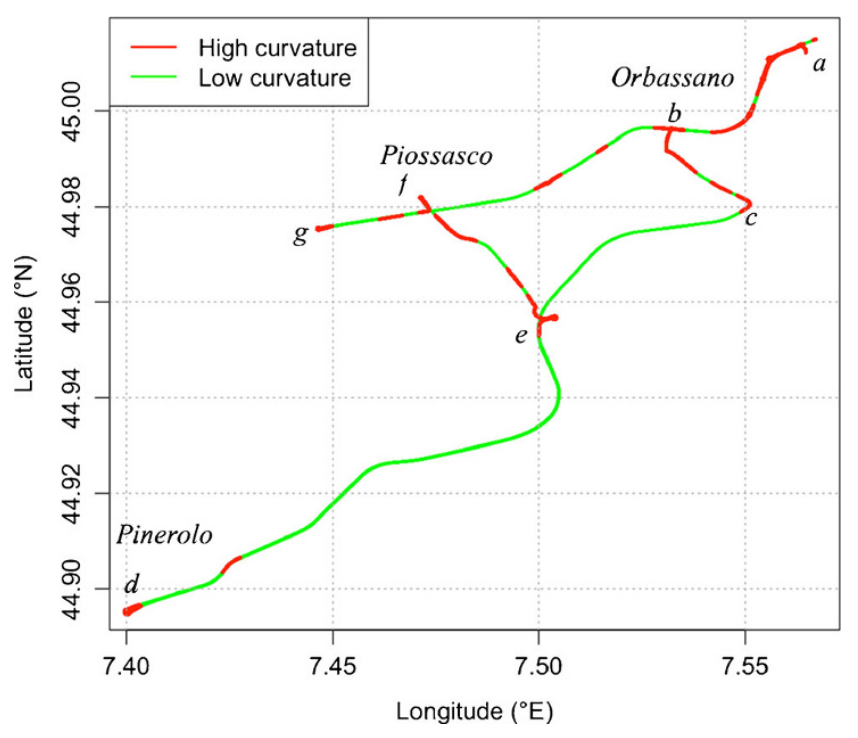

Fig. 1 Test Route. An online version is available [47] 
Table 1 Test track main characteristics

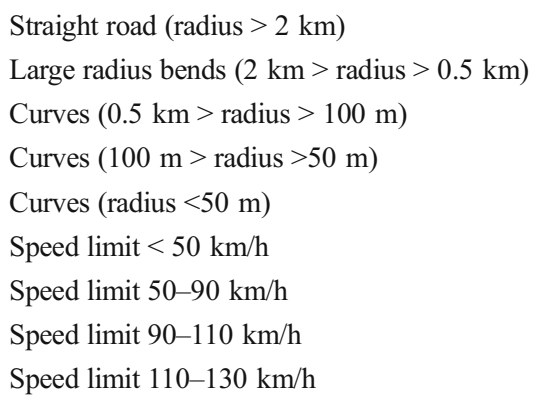

$27.8 \mathrm{~km}$

$20.6 \mathrm{~km}$

$2.7 \mathrm{~km}$

$912 \mathrm{~m}$

$821 \mathrm{~m}$

$7.2 \mathrm{~km}$

$10.3 \mathrm{~km}$

$5.4 \mathrm{~km}$

$29.5 \mathrm{~km}$
The co-driver is an artificial cognitive system, which implements the mirroring mechanism posited in above cited theories on human empathy [1-6]. The co-driver can be described as a human-like tutor, who compares the driver behavior to own-generated optimal behaviors. The mirroring of observed behaviors lets the co-driver understand the intentions of the human driver (ruling out intentions that do not match the observed behavior). It may thus suggest corrections for fixing or improving maneuvers that are improperly executed by the human driver, while preserving his original intention. This function has been named Continuous Support [8], and it essentially uses the co-driver as a peer.

The test vehicle is equipped with a perception platform collecting data from a number of sensors:

- one laser scanner, one long-range forward-looking radar, and one camera are combined to provide a description of front objects;

- a camera-based lane recognition system is combined with digital maps and GPS signals to produce a description of the surrounding road geometry;

- side-mounted ultrasonic sensors, and rear-looking shortrange radar detect side and rear obstacles;

- finally, on-board sensors collect information about ego motion.

For the purpose of this work, only the data provided by the latter system - which are listed in Table 2-are of interest. These signals are collected with a regular sampling rate of $10 \mathrm{~ms}$, then sent to the perception platform. In turn, the perception platform produces a description of the ego-state, the environment, and obstacles, which is sent to the co-driver, with an irregular refresh rate of approximately $100 \mathrm{~ms}$. The signals are preprocessed on the $10 \mathrm{~ms}$ side: all signals are filtered with a first order low-pass digital filter with a cut-off frequency of $20 \mathrm{~Hz}$, except the steering rate signal, which is cut at $5 \mathrm{~Hz}$.

For the purposes of the present work, a subset of the available signals is extracted from the co-driver logs according to the entries reported in Table 2. Given the average refresh rate of $100 \mathrm{~ms}$, there are approximately 25,000 samples for
Table 2 On-board ego-motion signals

\begin{tabular}{lll}
\hline Signal & Description & Units \\
\hline $\begin{array}{l}\text { ECUtime } \\
\text { cycleNumber }\end{array}$ & ECU time & $\mathrm{ms}$ \\
VLgtFild & $\begin{array}{l}\text { Perception Platform cycle count } \\
\text { Filtered longitudinal velocity } \\
\text { from odometer }\end{array}$ & $\mathrm{m} / \mathrm{s}$ \\
ALgtFild & $\begin{array}{l}\text { Filtered longitudinal acceleration } \\
\text { ALatFild }\end{array}$ & $\mathrm{m} / \mathrm{s}^{2}$ \\
YawRateFild & Filtered lateral acceleration & $\mathrm{m} / \mathrm{s}^{2}$ \\
SteerWhlAg & Filtered yaw rate & $\mathrm{rad} / \mathrm{s}$ \\
SteerWhlAgSpd & Filtered steering wheel angle & $\mathrm{rad}$ \\
SteerTorque & $\begin{array}{l}\text { Filtered steering wheel rate } \\
\text { GasPedPos }\end{array}$ & $\mathrm{rad} / \mathrm{s}$ \\
BrakePedPos & Filtered steering column torque & $\mathrm{Nm}$ \\
BrakePedalSwitchNCSts & Filtered gas pedal position & $\%$ \\
ActGear & $\begin{array}{l}\text { Filtered master cylinder pressure } \\
\text { Brake pedal pressed }\end{array}$ & $\mathrm{Nm}$ \\
IndTurnComm & Actual gear & \\
egoLatitude & Indicator turn command & \\
egoLongitude & (0: off, 1: left, 2: right) & \\
GPSspeed & GPS latitude & $\mathrm{deg}$ \\
GPScourse & GPS longitude & $\mathrm{deg}$ \\
& GPS velocity & $\mathrm{m} / \mathrm{s}$ \\
\hline & GPS velocity direction & $\mathrm{rad}$ \\
& (clockwise from north) & \\
\hline
\end{tabular}

every signal and for every trip (i.e. a database of nearly 1.2 million samples per signal).

\subsection{Subjects}

Twenty-four subjects drove the test track twice. For each driver, in one run the Continuous Support system was active but silent, in the other run the system-human interactions were active. Half of the subjects, randomly selected, drove first with the co-driver system turned on, the other half began with the system turned off.

Statistical data about test subjects and test modality are given in Table 3.

\section{Acceleration}

\subsection{Lateral acceleration, curvature and speed}

Figure 2 gives a comprehensive view of the lateral acceleration versus speed for all runs and subjects.

Levinson [37], page 58, recommends $a_{\text {lat }}=(k / v)^{2}$ with $k=$ $36 \mathrm{~m}^{3 / 2} / \mathrm{s}^{2}$, as a criterion for the accepted lateral acceleration, $a_{\text {lat }}$, as function of speed, $v$, for the average driver. A second criterion for $85^{\text {th }}$ percentile driver is given as $k=42 \mathrm{~m}^{3 / 2} / \mathrm{s}^{2}$. A maximum value for lateral acceleration also holds, which is 
Table 3 Drivers data

\begin{tabular}{cllllll}
\hline Driver & Gender & $\begin{array}{l}\text { Age } \\
\text { class }^{\mathrm{a}}\end{array}$ & $\begin{array}{l}\text { Km/year } \\
\text { driven }\end{array}$ & $\begin{array}{l}\text { Road } \\
\text { condition }\end{array}$ & Co-Driver & Driving \\
\hline exp'ce. (y) & & & & & \\
1 & $\mathrm{f}$ & 3 & 10,000 & $\mathrm{w} \mathrm{d}$ & 01 & 28 \\
2 & $\mathrm{~m}$ & 3 & 20,000 & $\mathrm{w} \mathrm{w}$ & 10 & 25 \\
3 & $\mathrm{~m}$ & 2 & 30,000 & $\mathrm{w} \mathrm{w}$ & 10 & 14 \\
5 & $\mathrm{f}$ & 2 & 8,500 & $\mathrm{~d} \mathrm{~d}$ & 01 & 18 \\
6 & $\mathrm{~m}$ & 2 & 15,000 & $\mathrm{~d} \mathrm{~d}$ & 01 & 23 \\
7 & $\mathrm{~m}$ & 3 & 15,000 & $\mathrm{~d} \mathrm{~d}$ & 10 & 20 \\
8 & $\mathrm{f}$ & 2 & 15,000 & $\mathrm{~d} \mathrm{~d}$ & 01 & 23 \\
9 & $\mathrm{~m}$ & 2 & 12,000 & $\mathrm{~d} \mathrm{~d}$ & 01 & 25 \\
10 & $\mathrm{f}$ & 2 & 15,000 & $\mathrm{~d} \mathrm{~d}$ & 01 & 18 \\
11 & $\mathrm{~m}$ & 3 & 16,000 & $\mathrm{~d} \mathrm{~d}$ & 10 & 32 \\
12 & $\mathrm{~m}$ & 3 & 20,000 & $\mathrm{~d} \mathrm{w}$ & 01 & 31 \\
13 & $\mathrm{~m}$ & 2 & 35,000 & $\mathrm{~d} \mathrm{~d}$ & 10 & 25 \\
14 & $\mathrm{f}$ & 3 & 10,000 & $\mathrm{~d} \mathrm{~d}$ & 10 & 30 \\
15 & $\mathrm{~m}$ & 2 & 30,000 & $\mathrm{~d} \mathrm{~d}$ & 01 & 25 \\
17 & $\mathrm{f}$ & 2 & 22,000 & $\mathrm{~d} \mathrm{~d}$ & 01 & 13 \\
18 & $\mathrm{f}$ & 2 & 30,000 & $\mathrm{~d} \mathrm{~d}$ & 10 & 12 \\
19 & $\mathrm{f}$ & 2 & 3,000 & $\mathrm{~d} \mathrm{~d}$ & 10 & 19 \\
20 & $\mathrm{f}$ & 2 & 5,000 & $\mathrm{~d} \mathrm{~d}$ & 10 & 9 \\
21 & $\mathrm{f}$ & 2 & 15,000 & $\mathrm{~d} \mathrm{~d}$ & 10 & 19 \\
22 & $\mathrm{~m}$ & 3 & 12,000 & $\mathrm{w} \mathrm{d}$ & 10 & 37 \\
23 & $\mathrm{~m}$ & 2 & 15,000 & $\mathrm{~d} \mathrm{~d}$ & 01 & 17 \\
24 & $\mathrm{f}$ & 2 & 20,000 & $\mathrm{~d} \mathrm{~d}$ & 01 & 11 \\
\hline & & & & & \\
\hline
\end{tabular}

a: age 25-44 years, 3: age 45-64 years

${ }^{\mathrm{b}} \mathrm{d}$ : dry road; w: wet road

${ }^{\mathrm{c}} 0$ : co-driver silent; 1 : co-driver is active

$a_{\max }=0.4 \mathrm{~g}\left(\sim 3.92 \mathrm{~m} / \mathrm{s}^{2}\right)$. Levinson's recommendations are, in turn, based on the Battelle study [48].

The dashed line in Fig. 2 represents a modified Levinson's criterion that proves to better fit the envelope of lateral accelerations presented in this study. It has the following equation:

$a_{\text {lat }}=\frac{a_{0}}{\sqrt{\left(1-\left(v / v_{0}\right)^{2}\right)^{2}+2\left(v / v_{0}\right)^{2}}}$

with $a_{0}=5.22, v_{0}=14.84$.

At a first glance, however, both criteria fail to describe driver lateral accelerations at high speed. To clarify this finding, the parabolas representing road bends of radius $600 \mathrm{~m}$ and $10 \mathrm{~m}$, respectively, are also plotted on the same chart. If a vehicle were moving at constant curvature, its lateral acceleration ad different speeds would indeed fall onto a parabola on the $a$ vs. $v$ chart.

On the left side of the parabola corresponding to $10 \mathrm{~m}$ radius bends there are virtually no observations, because that is the minimum radius experienced in the test track. On the

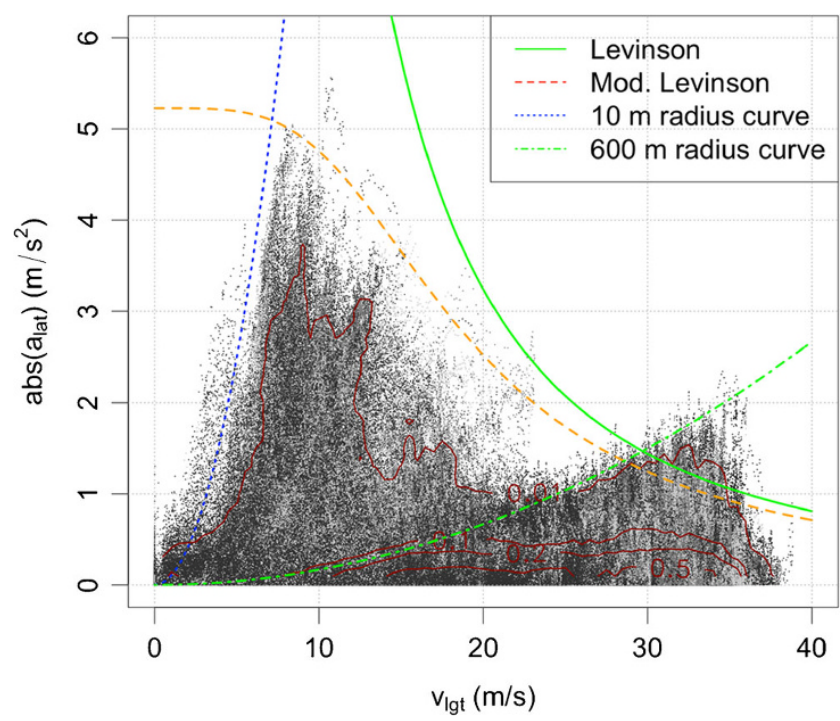

Fig. 2 Lateral acceleration versus speed for all runs and subjects. The curve labeled as "Levinson" is the Levinson's criterion

other hand, points laying under the parabola corresponding to $600 \mathrm{~m}$ radius bends represent the large- and very-large radius bends found along the motorway and along some part of extra-urban roads (Table 1).

These observations point to the conclusion that human drivers actually use (at least) two different driving modalities (i.e. two ECOM states), for motorway-like scenarios and for windier/slower roads. In the latter case, speed and curvature co-vary.

\section{1) The two-thirds power law}

The two-thirds power law refers to the experimental observation about the regularity and smoothness that is apparent in human and primates movements $[19,20]$. It was found that speed and curvature in hand movements are correlated, such as:

$v=\alpha \rho^{-1 / 3}$

That is, the velocity $v$ at which the path is traced (for example during handwriting) is proportional to the path curvature $\rho$, raised to $-1 / 3$. The exponent would be $-2 / 3$ if the left side of equation (2) were the angular speed, hence the "two-thirds power law" name.

The constant $\alpha$ may change for different classes of motor primitives. In fact, the two-thirds power law, initially discovered for hand movements, was later found to describe many other body movements such as foot trajectories, eye and speech movements and human locomotion [49].

Note that the Levinson's criterion would read as $v=\alpha^{\prime} \rho^{-1 / 4}$ i.e., with an exponent $-1 / 4$ very close to the two-thirds power law. Note also that in human locomotion exponents between $-1 / 3$ and $-1 / 4$ are observed in place of the strict $-1 / 3$ of the original law [49]. 
According to recent studies $[15,18,21]$, the two-thirds power law is considered as a consequence of optimality principles that generate human motion, and in particular of the minimum variance principle. In short, in order to improve movement accuracy, while preserving average speed, it is convenient to increase speed in straighter arcs and reduce it along curvier ones. Again, as above noted, different motor primitives may have different $\alpha$.

To explain the inverse relation between speed and curvature observed in driving, Winsum and Godthelp [32] proposed that speed modulation aims at minimizing the effects of steering errors. Interestingly this is the same concept of the minimum variance principle. Reymond and others [36] reformulated the idea in terms of safety margins producing a theoretical equation that they found to fit data collected on a circuit with 7 drivers.

However, Reymond's equation and data do not agree with data found in this study: firstly, they predict and observe maximum lateral acceleration in the range of $7-8 \mathrm{~m} / \mathrm{s}^{2}$ at low speed, whereas our data and data from Battelle are limited to $\sim 4 \mathrm{~m} / \mathrm{s}^{2}$. Secondly, they predict that lateral acceleration becomes exactly zero at approximately $35 \mathrm{~m} / \mathrm{s}$, which is contradicted by data reported in Fig. 2 .

The hypothesis that the two-thirds power law holds also for driving is hereafter assumed as a convenient way to interpret recorded data. The difference with human body movements is that now the brain is controlling a different plant, i.e. the vehicle. If the law is a byproduct of motor optimality principles, it is plausible that the very mechanism that produces the two-thirds law for the human body also produces something quite similar when controlling a vehicle.

To support this hypothesis, the Fig. 3 is presented, where local path radius is plotted versus speed in a log-log chart.

Red and black points represent the populations for data collected during trips with co-driver on and off, respectively. Also, the horizontal line at $300 \mathrm{~m}$ radius is drawn to

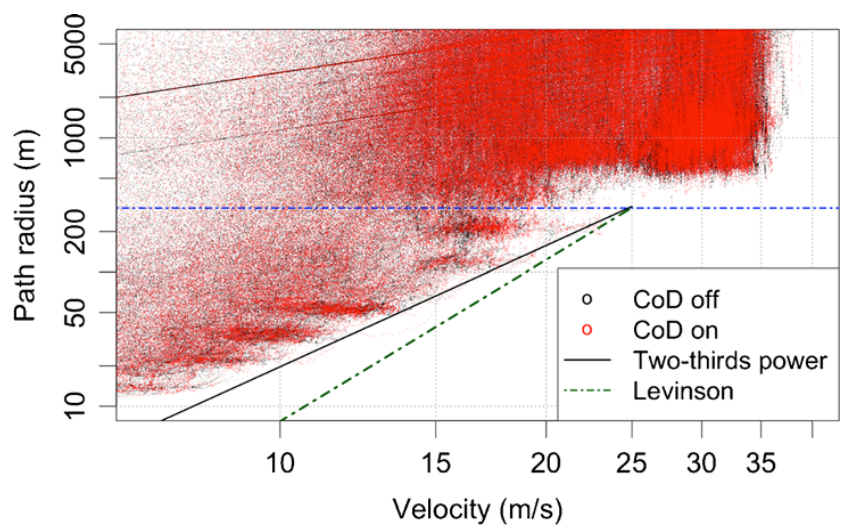

Fig. 3 Curvature versus velocity chart, in double log scales, shows that the two-thirds power law may describe the fastest maneuvers. Red points and black points were collected with co-driver on and off, respectively conveniently divide two regions, corresponding to roads of higher and lower curvature, respectively. These regions are indicated with different colors in Fig. 1 (more precisely, a map point is considered to belong to a low curvature segment if no radius of curvature smaller than $300 \mathrm{~m}$ has been or will be met in $10 \mathrm{~s}$ ). Roads that qualify as having low curvature with this definition are the motorway and some nearly straight segments with no roundabouts on the extra urban roads. High curvature roads are traits including or nearby curves and roundabouts.

The two-thirds power law, which accounts for 0.999 quantile of roads of high curvature only, is also plotted, both for the data population with co-driver on and off. The dotdashed line plots the Levinson's rule, which is clearly less fit to the experimental observations.

As a side note, few straight lines in the top left region of the $\log -\log$ chart are due to malfunctioning of the vehicle sensor system that was not updating the sole value of the instant yaw rate. These acquisition errors only affect the yaw rate channel, and for a subset of samples that collects less than $0.1 \%$ of the whole dataset.

Figure 3 provides the basis for a number of considerations. Firstly, it is worth noting that there are denser horizontal clouds of points at discrete radii, which are the most frequent radii in the track (curvature is not uniformly sampled as said in Table 1). The rightmost and topmost cloud is the motorway, with curvature radii greater than $600 \mathrm{~m}$ radius (except one short trait near Pinerolo).

Secondly, the two-thirds power law curve describes only the frontier of low clouds. There are points laying left to it. For example, let us consider the rather dense cloud at $50 \mathrm{~m}$ radius. It touches the two-thirds curve at about $14 \mathrm{~m} / \mathrm{s}$, but, left of it, there were recorded situations at which the same curve was driven at lower speed. In other words, the two-thirds power law holds for the fastest maneuvers, and describes situations in which speed must be modulated according to curvature, but for slower trajectories there is no longer such need to adapt speed to the curve. Consequently, one may argue that on the left of the curve there is a transition zone to constant speed motor primitives, and only at the clouds frontier the motor primitives follow (2).

Finally, the motorway scenarios (the topmost and rightmost cloud) are all of the constant speed type. They trespass the two-thirds law curve drawn in Fig. 3 (which holds for high curvature). It is here supposed that in motorways, where lanes are wider, the speed accuracy tradeoff is shifted in favor of speed, still withstanding law (2) but with a larger value of $\alpha$. This however could not be observed because nobody drove fast enough to need modulating the speed in motorway curves.

\section{2) Human lateral acceleration in curves}

Given the above considerations, the two-thirds law is here adopted to describe extreme maneuvers in roads with high 


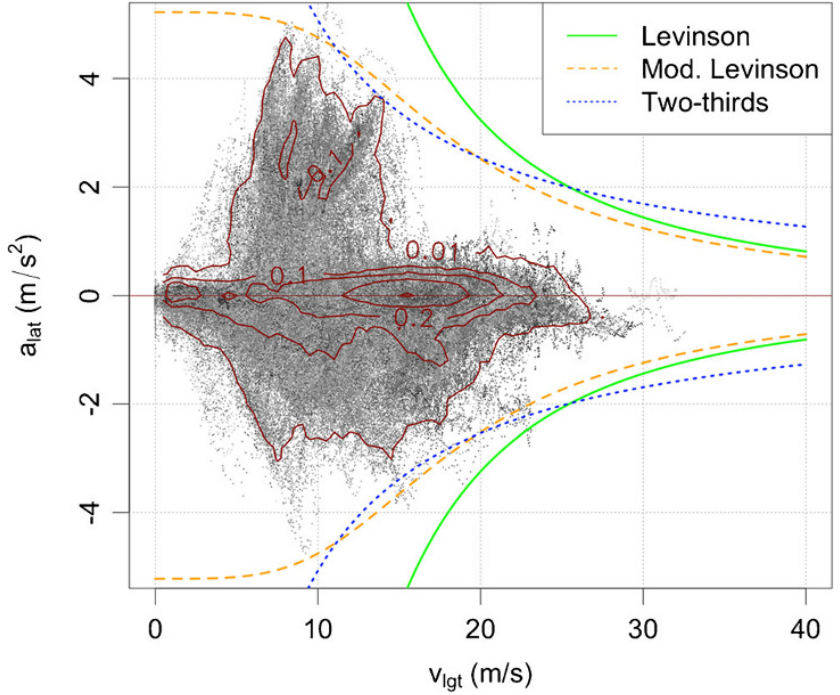

Fig. 4 Lateral acceleration versus speed in high-curvature roads. Speed is modulated according to curvature and acceleration is limited by (1) or (2)

curvature (Figs. 1 and 3). The coefficient representing the quantile 0.999 of data is:

$\alpha=3.70 \mathrm{~m}^{2 / 3} / \mathrm{s}$

It has to be remarked that, even if the above has been found with a user group of mixed characteristics, there still might be some skewing factor, such as the nationality (drivers are all Italians), the use of the very same car, or limitations in the scenarios (e.g., curvatures, road widths, etc.) covered by the test track.

Figure 4 presents the lateral accelerations for roads of high curvature. It is equivalent to Fig. 1, except that data of low curvature segments are stripped off and that the acceleration is shown with sign (positive accelerations are left curves). The

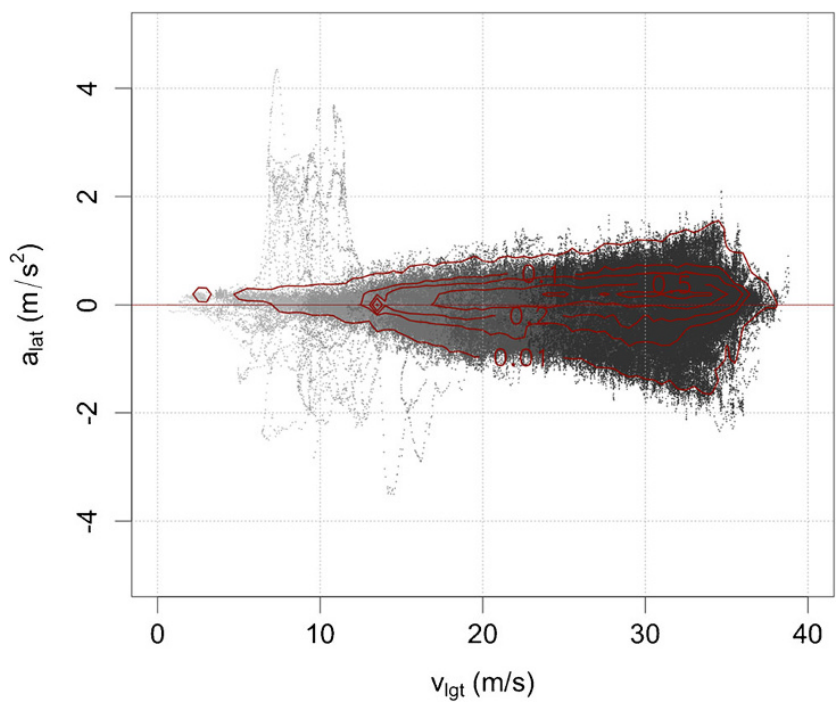

Fig. 5 Lateral acceleration vs. speed in roads of low curvature. Acceleration is proportional to the square of speed, meaning that driving occurs at constant velocity

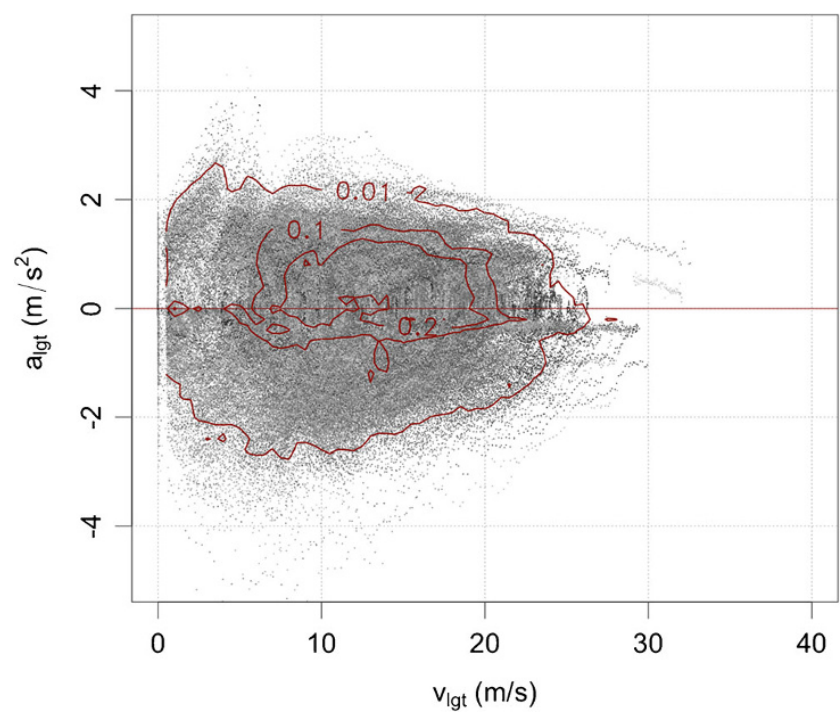

Fig. 6 Longitudinal acceleration versus speed in high-curvature scenarios

chart looks asymmetric because radii and frequencies of curves to the left are different than to the right. Most of the track is indeed driven in both directions, but ramps and roundabouts are asymmetric. In particular, all roundabouts are sharp left curves. The two-thirds power law, the Levinson, and the modified Levinson criteria are also shown.

Figure 5 is the same but for the roads of low curvature, and it shows that in this case the driving modality is constant speed.

\section{3) Human longitudinal acceleration}

Figures 6 and 7 show the longitudinal acceleration versus speed for high and low curvature roads, respectively. The whole data set (all drivers, all runs) is here considered.

Unlike lateral acceleration, longitudinal acceleration appears to be, at least at this global level, loosely related to

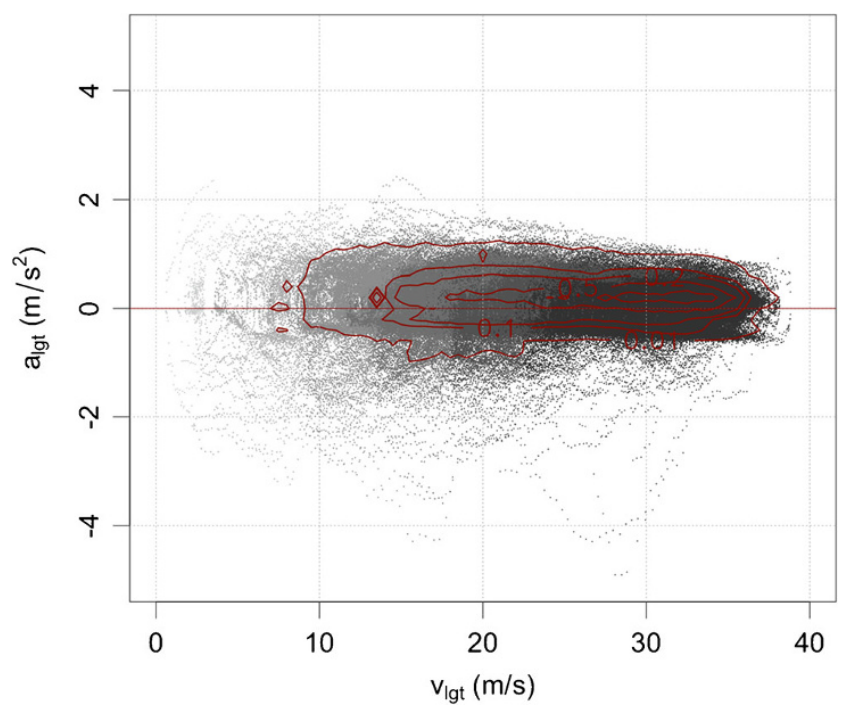

Fig. 7 Longitudinal acceleration versus speed in motorways and similar (low-curvature) scenarios 


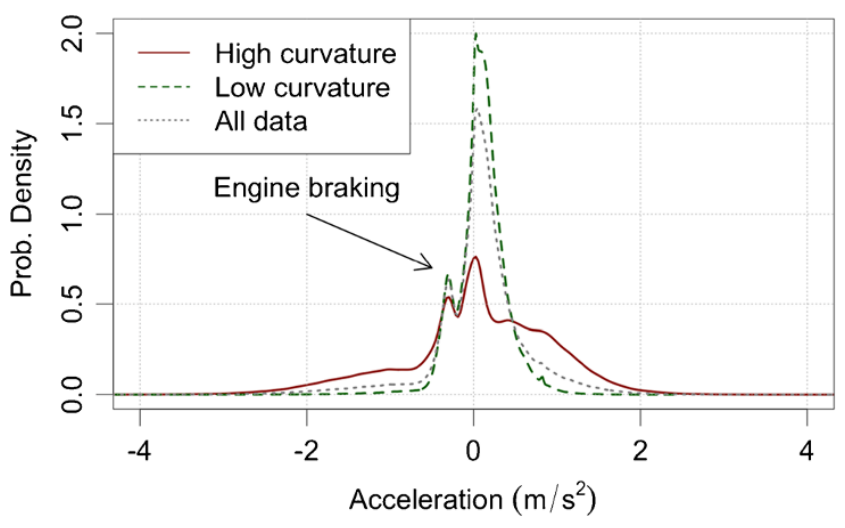

Fig. 8 Distribution kernel density estimate of longitudinal acceleration for all data (dotted line), for low-curvature roads (solid), and for highcurvature roads (dashed). The peak at about $-0.35 \mathrm{~m} / \mathrm{s}^{2}$ corresponds to motor brake action

speed. The causes for longitudinal acceleration may be curves (need to adapt speed) or may be others (e.g. speed limits, obstacles, traffic etc.). Figures 6 and 7 give an overall picture of longitudinal speed changes with no distinction for the cause.

The maximum forward acceleration declines slowly with speed. For the high-curvature scenario, the longitudinal acceleration is less than about $2 \mathrm{~m} / \mathrm{s}^{2}$ at 0.01 percentiles. For motorways, and low curvature roads in general, the maximum observed acceleration is about $1 \mathrm{~m} / \mathrm{s}^{2}$ at 0.01 percentiles.

Braking deceleration also looks fairly constant. For highcurvature scenarios (Fig. 6) it is about $-2 \mathrm{~m} / \mathrm{s}^{2}$ at 0.01 percentiles, but there is a tail of rare events, where greater decelerations may be observed. Figure 7 shows a neat boundary corresponding to the gas pedal completely released and no brake (engine braking condition). This state happens frequently enough to produce a denser region of points in the chart of Fig. 7, and in the right part of Fig. 6 , at around $-0.5 \mathrm{~m} / \mathrm{s}^{2}$, and decreasing with speed according to the aerodynamic drag.
Below that condition, decelerations result by true braking action.

Figure 8 shows the distribution density of longitudinal acceleration with no distinction for speed (in force of the weak speed dependency above discussed). The two curves are for the two types of scenarios.

Table 4 gives an analysis of the longitudinal accelerations in a form comparable to [40]. The row for the complete dataset is comparable to Table 4 in the cited paper. Note that data for the complete set is a weighted average of different driving conditions (e.g., high and low curvature regions), which are quite different. Note that Table 4 also lists the observed accelerations for the same ranges of speed listed in [40], Table 5 .

According to [40], $90 \%$ of observed longitudinal accelerations for the complete dataset fall into the $-1.03 \div 0.91 \mathrm{~m} / \mathrm{s}^{2}$, whereas data of this paper indicate the interval $-0.89 \div 0.96 \mathrm{~m} / \mathrm{s}^{2}$. Braking decelerations are also reported in Table 4 to be compared to Table 8 of the same paper. A fair agreement between these data can be observed, although the different testing conditions, vehicle type, nationality, data set dimension, and test track type can easily explain the differences among the distributions, even more when considering the marked difference in distribution when classifying different track segments on the basis of the local curvature.

\subsection{The g-g diagram}

Figures 3, 4, 5, 6, and 7 do not reveal existing correlations between longitudinal and lateral acceleration. Indeed, maneuvers that withstand the two-thirds power law have a distinctive pattern: decelerate-steer-accelerate. Such pattern is otherwise not observed in constant speed maneuvers.

To reveal how lateral and longitudinal accelerations are correlated, a useful chart is the g-g diagram [28, 41-43].
Table 4 Analysis of longitudinal accelerations

\begin{tabular}{llllllll}
\hline Percentile & Min. & $5 \%$ & $25 \%$ & $50 \%$ & $75 \%$ & $95 \%$ & Max. \\
\hline Accel'n $\left(\mathrm{m} / \mathrm{s}^{2}\right)$ & & & & & & & \\
$\quad$ - High-curvature & -8.87 & -1.63 & -0.35 & 0.06 & 0.65 & 1.38 & 4.43 \\
- Low curvature & -4.90 & -0.38 & -0.05 & 0.09 & 0.24 & 0.56 & 2.42 \\
- All data & $-\mathbf{8 . 8 7}$ & $\mathbf{- 0 . 8 9}$ & $-\mathbf{0 . 1 2}$ & $\mathbf{0 . 0 9}$ & $\mathbf{0 . 3 0}$ & $\mathbf{0 . 9 6}$ & $\mathbf{4 . 4 3}$ \\
- 0-40 km/h & -8.87 & -1.79 & -0.39 & 0.04 & 0.60 & 1.49 & 4.43 \\
- 40-70 km/h & -4.77 & -1.24 & -0.26 & 0.11 & 0.47 & 1.13 & 3.25 \\
- Over 70 km/h & -4.90 & -0.38 & -0.05 & 0.09 & 0.23 & 0.55 & 2.06 \\
Braking $\left(\mathrm{m} / \mathrm{s}^{2}\right)$ & & & & & & & \\
- All data & $-\mathbf{8 . 8 7}$ & $-\mathbf{1 . 6 1}$ & $-\mathbf{0 . 4 4}$ & $-\mathbf{0 . 2 6}$ & $-\mathbf{0 . 0 9}$ & $-\mathbf{0 . 0 1}$ & $\mathbf{0}$ \\
- Under $40 \mathrm{~km} / \mathrm{h}$ & -8.87 & -2.22 & -1.14 & -0.47 & -0.11 & -0.02 & 0 \\
- 40-70 km/h & -4.77 & -1.72 & -0.72 & -0.32 & -0.18 & -0.03 & 0 \\
- Over 70 km/h & -4.90 & -0.54 & -0.32 & -0.16 & -0.06 & -0.01 & 0 \\
\hline
\end{tabular}


Table 5 Percentiles of user descriptors

\begin{tabular}{lrrr}
\hline & $5 \%$ & $50 \%$ & $95 \%$ \\
\hline$\left.\alpha . \mathrm{m}^{2 / 3} / \mathrm{s}\right)$ & 3.06 & 3.34 & 3.60 \\
$\mathrm{a}\left(\mathrm{m} / \mathrm{s}^{2}\right)$ & 3.66 & 4.49 & 5.53 \\
$\mathrm{~b}\left(\mathrm{~m} / \mathrm{s}^{2}\right)$ & 2.42 & 3.10 & 4.12 \\
$\mathrm{c}\left(\mathrm{m} / \mathrm{s}^{2}\right)$ & -5.27 & -3.86 & -3.09 \\
\hline
\end{tabular}

Figures 9 and 10 show the g-g diagram for the high and low curvature scenarios, respectively.

Figure 9, for high curvature low speed scenarios, looks noticeably asymmetric, due to asymmetry of the test track and due to the presence of roundabouts. In Fig. 9, positive lateral accelerations mean leftwards curves, and roundabouts are all leftwards. In particular, the island in the middle of the right half of the chart centered at $\sim 2.5 \mathrm{~m} / \mathrm{s}^{2}$ is caused by roundabouts.

Percentile contour plots reveal interesting features. Lets first consider the inner contour, corresponding to $99.9 \%$ of driving time ( 0.1 percentiles label). This shows a distinct mushroom shaped pattern. The mushroom stipe corresponds to braking. At 0.1 percentiles ( $99.9 \%$ of driving time) braking occurs between approximately $-0.5 \mathrm{~m} / \mathrm{s}^{2}$ and $-2 \mathrm{~m} / \mathrm{s}^{2}$. The corresponding lateral acceleration is smaller than $0.5 \mathrm{~m} / \mathrm{s}^{2}$. This means that for most of driving, braking occurs without significant lateral accelerations.

The mushroom cap, from $-0.5 \mathrm{~m} / \mathrm{s}^{2}$ to $2 \mathrm{~m} / \mathrm{s}^{2}$ longitudinal acceleration, occurs when the accelerator is used and brake is not. In this modality, longitudinal accelerations are combined with much higher lateral accelerations, up to about $4 \mathrm{~m} / \mathrm{s}^{2}$, especially in roundabouts. During $99.9 \%$ of driving in these conditions, accelerator is used in combination with steering, unlike braking, which as said occurs without significant steering.

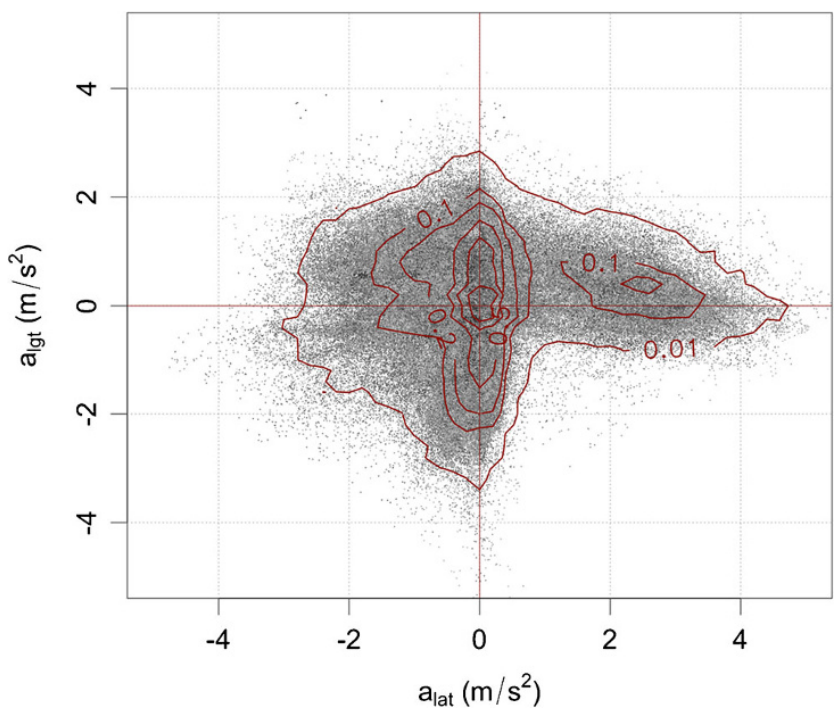

Fig. 9 g-g diagram for high-curvature scenarios

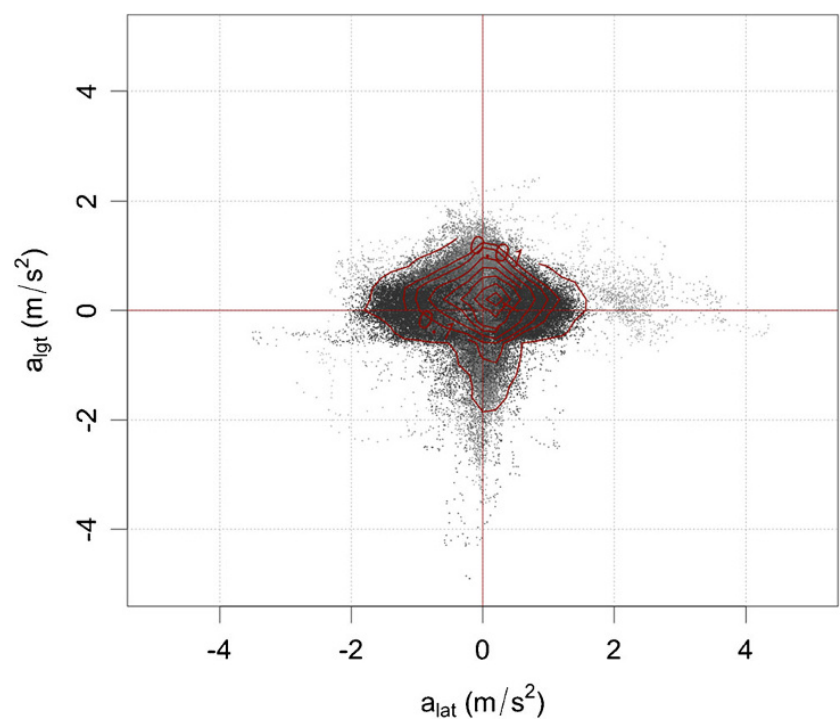

Fig. 10 g-g diagram in low-curvature (high speed) scenarios

Following the ECOM or Michon frameworks, we may argue the existence of different behaviors and/or motor primitives at the monitoring/regulating/tracking levels of the ECOM architecture (or Michon's operational/tactical levels). One uses the brake without significant lateral acceleration. Another combines the use of the accelerator and the steering wheel for speed-curvature coordination. This latter includes as a sub-modality, in which the accelerator is fully released (i.e. saturation of deceleration, or engine braking) that is used so often to be noticeable. As a consequence, speed modulation in curvy roads is limited by engine braking saturation, and produces the mushroom shapes of the g-g diagrams (one

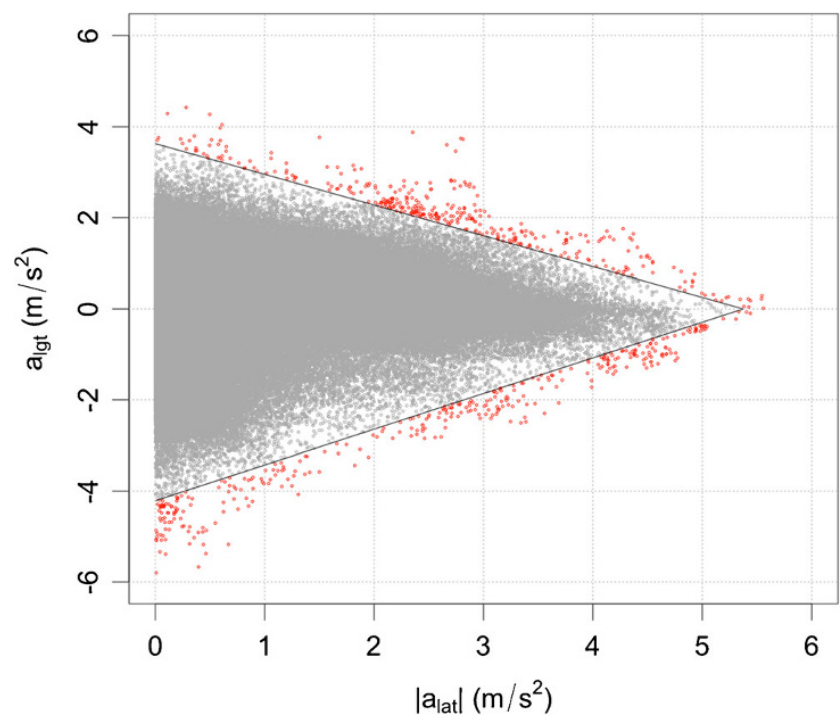

Fig. 11 Parameterization of driver g-g diagrams for the whole dataset (lateral acceleration is in modulus). The black triangle represents maximum accelerations $a, b$, and $c$, while red circles are points that remain outside the fitted triangle (see Section IV) 
Fig. 12 Typical trajectories in the g-g diagram for various maneuver types. From top to bottom: roundabouts (row 1 and 2), motorways (row 3 ), right bends or right-left-right bend sequences (row 4), sudden brake maneuvers with no steering (row 5), combined braking with right steering (row 6)
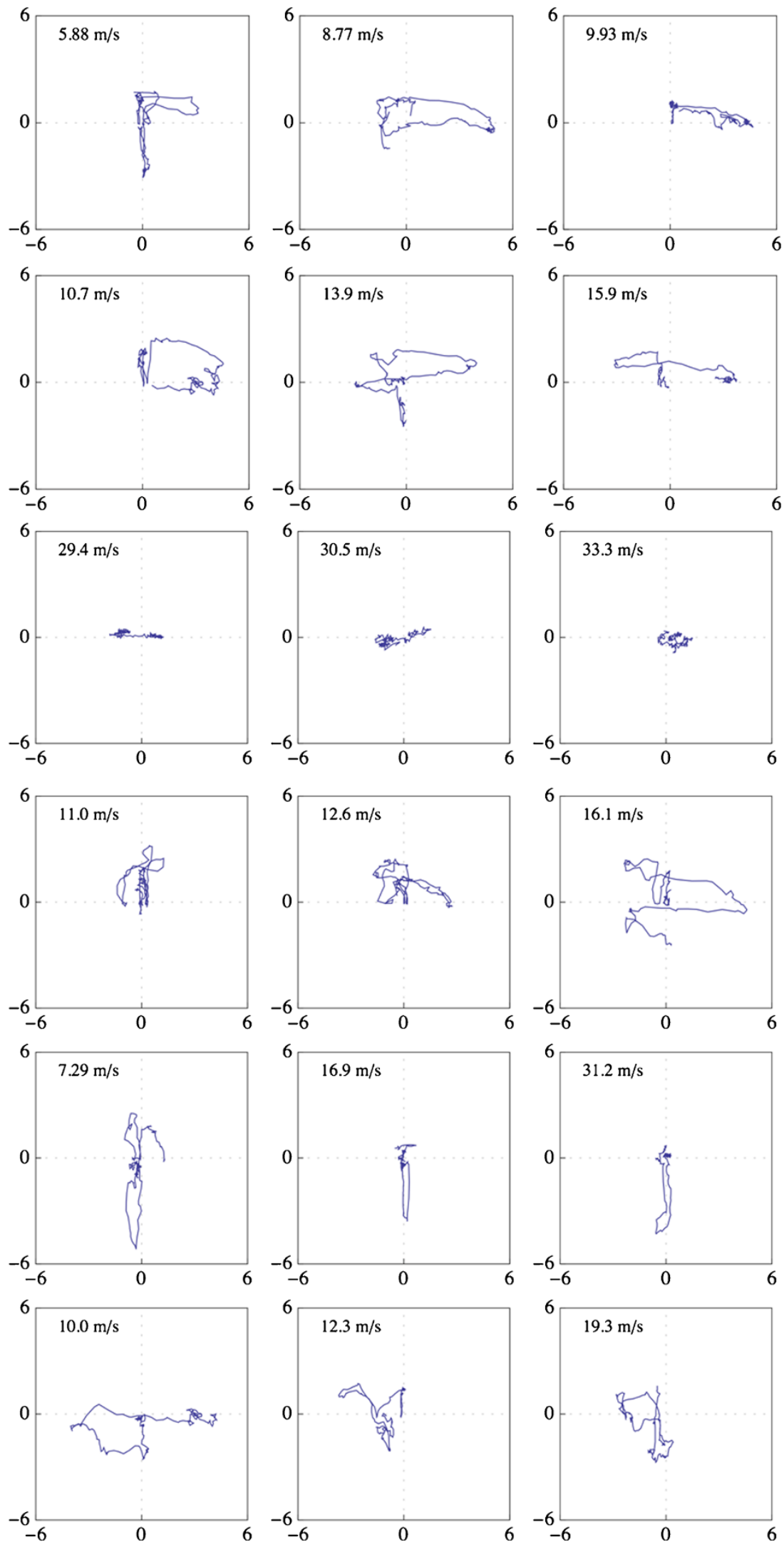

may argue that in electric vehicles, in which the electric engine may also brake, this shape should not be observed).
Let us now consider the points between 0.1 and 0.01 percentiles, or $0.09 \%$ of driving time (i.e. what lie between 
$99.9 \%$ and $99.99 \%$ ). These are less frequent events, which however take place several times in a travel. For these, the mushroom shape turns into a more rounded shape, in particular for the left part of Fig. 9, which represents curvy roads without roundabouts. These are typical brake-steer-accelerate maneuvers that are produced close to the region of validity of the two-thirds power law, i.e. when speed and curvature must co-vary. However, the right part of Fig. 9 shows that the way in which speed and curvature co-vary along roundabouts is different. This can be explained with the fact that yield signs typically precede the entrance in roundabouts. Thus, before entrance there can be no co-variation, and the mushroom stipe shape is preserved on the right parte of Fig. 9.

Even if more rare, there thus exist behaviors, in which brake and steer are combined.

Lets now consider the low-curvature high-speed roads (Fig. 10). Firstly it can be observed that the mushroom is more symmetric than in the previous case. Separation between the mushroom cap (use of accelerator only) and its stipe (braking) is also clearer. Moreover, a faint cloud of points representing the engine braking condition is also visible.

By looking at the mushroom stipe, it is apparent that, during braking, very little lateral acceleration is used. Conversely, when considering the mushroom cap, its diamond shape suggests that co-variation of lateral and longitudinal acceleration is linear in the acceleration phase. Such a linear relationship may indeed be noticed, although less clearly, in Fig. 9, where the mushroom cap at 0.01 percentiles is fairly straight on the right side only, whereas it is truncated on the left side.

The characteristic shape suggests a method to parameterize the g-g diagram by means of a diamond shape, with $a, b$, and $c$ representing the maximum longitudinal, lateral and braking accelerations as shown in Fig. 11 (see details in Sec. IV). In facts, if the road were specular, i.e. the sign of curves, were inverted, observations would have the opposite signs in lateral accelerations, and would thus fill the entire diamond when combined with the non-specular paths. In other words, the hypothesis is that humans have isotropic motor primitives in both directions and would produce specular accelerations for specular paths.

Figure 12 shows the typical g-g patterns for various types of maneuvers. The first two rows are a left turn at an intersection (top left) and along roundabouts, which tend to be negotiated with braking disjoined from steering on enter, and combined acceleration-steering on exit. The last two charts in the second row also show the right acceleration peak that precedes the entrance link. The third row is a typical motorway scenario, where lateral accelerations may occur without any speed adaptation. The fourth row depicts combined right steering and acceleration and more complex maneuvers. The fifth row shows braking maneuvers occurring with no steering. Finally, the sixth row shows combined right steering and braking. Gearshifts sometimes perturb these patterns.

Of all the cases depicted in Fig. 12, rows 1, 2, 4, and 6 are maneuvers that use combined longitudinal and lateral acceleration. The remaining rows represent unrelated longitudinal or lateral maneuvering.

Figure 13 shows how g-g diagrams change with speed. At very low speed $(0 \div 5 \mathrm{~m} / \mathrm{s}$, top left) there is virtually no lateral control. In the urban speed range $(5 \div 15 \mathrm{~m} / \mathrm{s}$, top center) the g$\mathrm{g}$ diagram has its maximum extension. It then gradually shrinks, loosing the mushroom shape at about $25 \mathrm{~m} / \mathrm{s}$, after which the diagram flattens, with growing lateral accelerations.

\section{User descriptors}

In order to investigate the effects of various extrinsic and intrinsic factors on the driving style, some quantitative indicators, or descriptors, synthetically representing each trip,
Fig. 13 g-g diagram dependency on speed
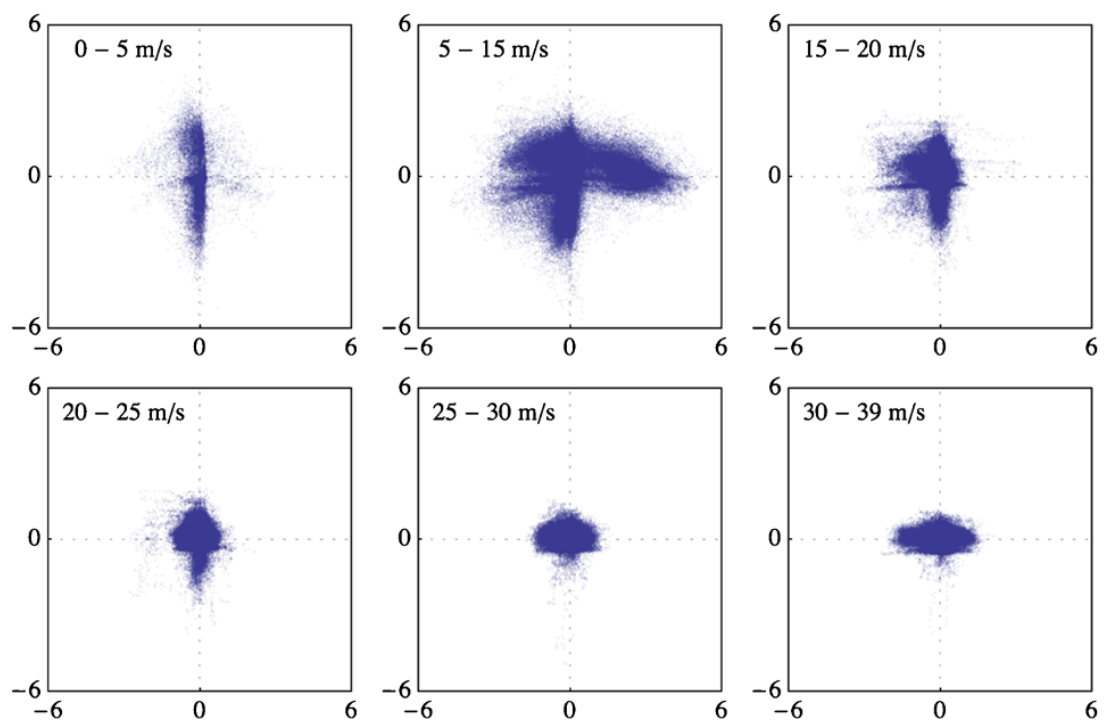

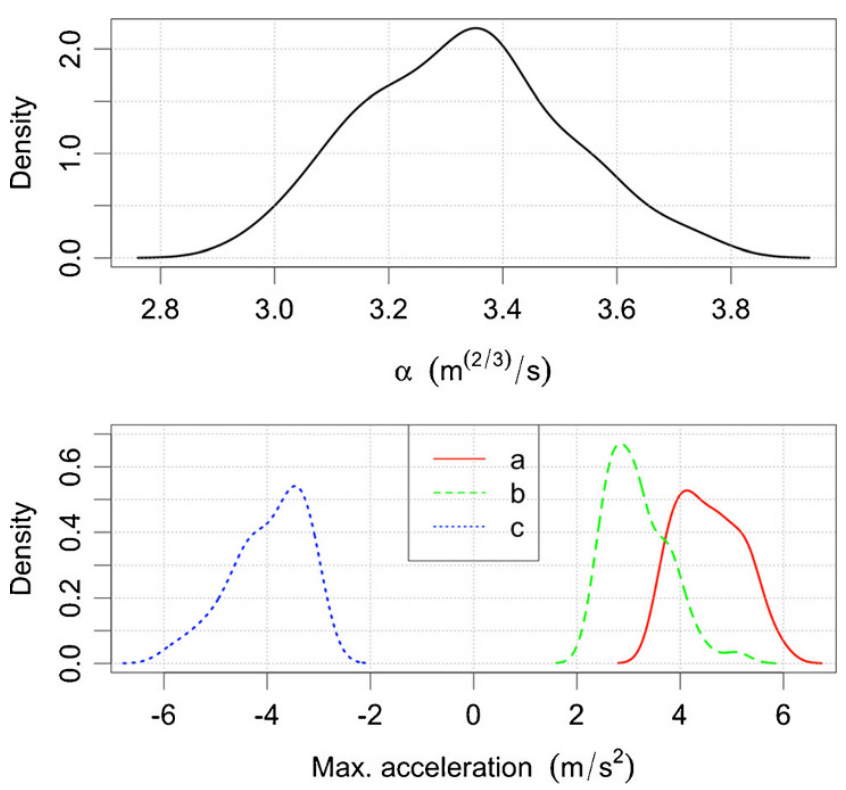

Fig. 14 Distribution kernel density estimates for $\alpha$ (top) and for maximum accelerations $a$ (lateral), $b$ (forward), and $c$ (braking) (bottom) have to be defined. From the point of view of users (or drivers), factors can be extrinsic (co-driver Continuous Support on/off state, road conditions, day/night) or intrinsic (gender, experience, car type usually driven, etc.)

User descriptors have been defined on the basis of the twothirds power law (the $\alpha$ coefficient), and on the basis of the maximum accelerations (forward $b$, backward $c$, and modulus of lateral $a$.)

Per each individual trip (two trips per user), the $\alpha$ coefficient has been calculated as the 0.999 quantile of the distribution of $v$ $\rho^{1 / 3}$ on data collected during high-curvature roads, where $v$ and $\rho$ are the velocity and the curvature, respectively. Data acquired at a velocity less than $1 \mathrm{~m} / \mathrm{s}$ are discarded, and curvature data are calculated as the ratio between the yaw rate and velocity.

The calculation of the maximum acceleration has been assessed in a slightly more complicated way. As above discussed (see Fig. 11) the $a_{\text {lgt }}$ vs. $\left|a_{\text {lat }}\right|$ chart is characterized by a rhomboid shape with a maximum lateral acceleration $a$, a maximum forward acceleration $b$, and a maximum backward (braking) acceleration $c$. Per each individual trip (two trips per
Table 6 Analysis variance (ANOVA)

\begin{tabular}{llllll}
\hline Response: $\boldsymbol{\alpha}$ & $\mathrm{Df}$ & Sum Sq & Mean Sq & F value & $\operatorname{Pr}(>\mathrm{F})$ \\
Gender & 1 & 0.00414 & 0.004136 & 0.6099 & 0.4431 \\
Experience & 6 & 0.34011 & 0.056685 & 8.3581 & $8.589 \mathrm{e}-05$ \\
Driver & 17 & 0.96437 & 0.056728 & 8.3645 & $4.643 \mathrm{e}-06$ \\
Co-Driver & 1 & 0.00477 & 0.004772 & 0.7036 & 0.4106 \\
Road Cond. & 1 & 0.00020 & 0.000203 & 0.0299 & 0.8643 \\
Residuals & 22 & 0.14920 & 0.006782 & & \\
Response: $\boldsymbol{a}$ & $\mathrm{Df}$ & Sum Sq & Mean Sq & F value & $\operatorname{Pr}(>\mathrm{F})$ \\
Gender & 1 & 0.0022 & 0.00223 & 0.0274 & 0.8700 \\
Experience & 6 & 4.7374 & 0.78956 & 9.6972 & $2.901 \mathrm{e}-05$ \\
Driver & 17 & 12.2202 & 0.71884 & 8.8286 & $2.906 \mathrm{e}-06$ \\
Co-Driver & 1 & 0.1090 & 0.10895 & 1.3381 & 0.2598 \\
Road Cond. & 1 & 0.0001 & 0.00007 & 0.0009 & 0.9764 \\
Residuals & 22 & 1.7913 & 0.08142 & & \\
Response: $\boldsymbol{b}$ & $\mathrm{Df}$ & $\mathrm{Sum} \mathrm{Sq}$ & Mean Sq & $\mathrm{F}$ value & $\operatorname{Pr}(>\mathrm{F})$ \\
Gender & 1 & 0.4773 & 0.47727 & 2.4683 & 0.13044 \\
Experience & 6 & 3.3640 & 0.56067 & 2.8997 & 0.03083 \\
Driver & 17 & 8.1785 & 0.48109 & 2.4881 & 0.02303 \\
Co-Driver & 1 & 0.7241 & 0.72405 & 3.7446 & 0.06594 \\
Road Cond. & 1 & 0.2639 & 0.26392 & 1.3649 & 0.25519 \\
Residuals & 22 & 4.2539 & 0.19336 & & $\operatorname{Pr}(>\mathrm{F})$ \\
Response: $\boldsymbol{c}$ & $\mathrm{Df}$ & $\mathrm{Sum} \mathrm{Sq}$ & Mean Sq & F value & 0.7706 \\
Gender & 1 & 0.0463 & 0.04633 & 0.0872 & 0.4088 \\
Experience & 6 & 3.4178 & 0.56963 & 1.0720 & 0.4124 \\
Driver & 17 & 9.9153 & 0.58325 & 1.0976 & 0.3652 \\
Co-Driver & 1 & 0.4543 & 0.45432 & 0.8549 & 0.9273 \\
Road Cond. & 1 & 0.0045 & 0.00453 & 0.0085 & \\
Residuals & 22 & 11.6908 & 0.53140 & & \\
\hline & & & & & \\
\hline
\end{tabular}


user) the full set of points $\left(a_{\mathrm{lgt}},\left|a_{\mathrm{lat}}\right|\right)$ has been used to find the values $a, b$, and $c$ that minimize the objective function $f$ :

$f=\left|\frac{N}{n_{a b c}}+\frac{a(b-c)}{\max \left(a_{\text {lat }}\right)\left(\max \left(a_{\text {lgt }}\right)-\min \left(a_{\text {lgt }}\right)\right.}\right|$

where $N$ is the number of points and $n_{a b c}$ is the number of points inside the triangle with vertices $a, b, c$ (where $c<0$ ). In each case, the number of points that remain outside the triangle is assumed as an indicator of the goodness of fit.

Figure 14 reports the distribution of $\alpha$ in the upper chart, and of $a, b$, and $c$ in the lower chart, for the whole set of individual test trips. The distribution estimate for $\alpha$ shows a moderate skewness $(0.16)$, while skewness is more pronounced for $a(0.26), b(0.51)$, and particularly $c(-0.69)$.

The 5, 50, and 95 percentiles of the four descriptors are reported in Table 5 for reference.

Finally, Table 6 reports the result for the analysis of variance for a linear model relating the four descriptors depending on gender of the driver, driver identity, his/her driving experience (as multiples of 5 years), the road condition (dry of wet), and the co-driver Continuous Support function status (on or off). Interactions are neglected as suggested by data screening here omitted for the sake of brevity.

The only factors resulting significant on all the four descriptors are the driver experience and his/her individuality, with a less pronounced significance level on the maximum braking deceleration $c$. The gender is not significant, being replaced by the experience level, as already noted in one early study on lateral accelerations in bends [31]. The action of co-driver, in conclusion, has a limited significance level (p-value of $6.6 \%$ ) on the maximum acceleration $b$. This is not surprising, considering that the intervention of the co-driver is a relatively rare event and thus its effects may be masked when examining the whole set of driving data.

\section{Conclusion}

This paper presents experimental measurements of vehicle accelerations observed in human driving, and reveals the existence of several "behaviors". The term "behavior" is used here in the sense of complex human sensory-motor strategies, which can be interpreted by means the ECOM conceptual framework as higher-level perception-action loops that activate different distinct motor primitives.

One major discrimination is noticed between behaviors that occur at constant speed (such as when the radius of curvature is large enough that no speed adaptation is necessary, typically along motorways) and behaviors where speed co-varies with curvature.

The latter case fits the two-thirds power law, which describes the speed/curvature correlation of large class human body movements. This suggests the idea that the same timeaccuracy tradeoff that make speed and curvature co-vary for human body movements is also effective when humans are controlling vehicles.

As for pure longitudinal control, three sub-behaviors have been observed: 1) use of accelerator, 2) use of engine brake, and 3) use of brake.

As for driving in conditions that need combined lateral and longitudinal control two behaviors have been separated:. 1) the use of combined accelerator (including engine brake retarding) and steering wheel is regularly observed for maneuvers requiring less than approximately $3 \mathrm{~m} / \mathrm{s}^{2}$ longitudinal deceleration; 2) instead, when longitudinal decelerations greater than $3 \mathrm{~m} / \mathrm{s}^{2}$ are demanded, and true braking is thus needed, the braking action mostly occur separately from the steering action. When real braking is necessary, human drivers split control into two separate motor units in sequence: braking and then steering.

Comparing with the available literature, the novelty of the present work consists in the experimental evidence that the twothirds power law - that was originally proposed within the neuro-motor research fields to describe human body movements - also applies to humans' vehicle driving behaviors.

Additionally, thanks to a significantly larger dataset w.r.t. previous works, a clearer picture with greater resolution is obtained, and it is has been possible to perform statistical inference tests that point out how the driving expertise is by far the most significant factor that differentiates the driving styles (in the sense that longer experience at driving is usually matched by faster driving style.)

Overall the paper gives useful data to model the above driver behaviors within ADAS applications..

As for future possible developments, we make the hypothesis that both the speed-curvature co-variation at the level of motor primitives, and the adoption of diverse sensory-motor strategies (e.g. different sequences of motor units such as combined deceleration-steering versus braking-steering) aim at robust control, i.e., the minimum variance principle introduced by Harris and Wolpert

What is presented in this paper is thus a first analysis of the collected data, focused on acceleration. However, it is our intention to analyze the data to identify motor units and chaining of motor units (higher-level strategies), to form a basis for testing the "robust control" hypothesis above.

Acknowledgments The Authors whish to thank Clemens Kaufmann and Anna Persson (Lund University), and Fabio Tango (CRF Torino) for their contribution in the conduit of test with subjects. 
Open Access This article is distributed under the terms of the Creative Commons Attribution License which permits any use, distribution, and reproduction in any medium, provided the original author(s) and the source are credited.

\section{References}

1. Hesslow G (2012) The current status of the simulation theory of cognition. Brain Res 1428:71-79

2. Jeannerod M (2001) Neural simulation of action: a unifying mechanism for motor cognition. Neuroimage 14(1 Pt 2):S103S109

3. Hurley S (2008) The shared circuits model (SCM): how control, mirroring, and simulation can enable imitation, deliberation, and mindreading. Behav Brain Sci 31(1):1-22, discussion $22-58$

4. Demiris Y (2007) Prediction of intent in robotics and multi-agent systems. Cogn Process 8(3):151-158

5. Wolpert DM, Doya K, Kawato M (2003) A unifying computational framework for motor control and social interaction. Philos Trans R Soc Lond B Biol Sci 358(1431):593-602

6. Demiris Y, Khadhouri B (2006) Hierarchical attentive multiple models for execution and recognition of actions. Robot Auton Syst 54(5):361-369

7. "interactive - Accident avoidance by intervention for Intelligent Vehicles." [Online]. Available: http://www.interactive-ip.eu/. [Accessed: 30-May-2013]

8. Saroldi A, Tango F, Da Lio M, Biral F, Galvani M (2012) Implementation of a Co-driver for continuous support. In: 19th ITS World Congress, no. October, p. EU-00748

9. Da Lio M, Biral F, Galvani M, and Saroldi A (2012) Will intelligent vehicles evolve into human-peer robots? In: IEEE Intelligent Vehicles Symposium, Proceedings, pp. 304-309

10. Da Lio M, Bosetti P, Saroldi A (To be submitted) Artificial co-drivers as a universal enabling technology for future intelligent vehicles and transportation systems. IEEE Trans Intell Transp Syst

11. Todorov E (2004) Optimality principles in sensorimotor control. Nat Neurosci 7(9):907-915

12. Liu D, Todorov E (2007) Evidence for the flexible sensorimotor strategies predicted by optimal feedback control. J Neurosci Off J Soc Neurosci 27(35):9354-9368

13. Mitrovic D, Klanke S, Vijayakumar S (2010) Adaptive optimal feedback control with learned internal dynamics models. Robotics 264:65-84

14. Nagengast J, Braun DA, Wolpert DM (2009) Optimal control predicts human performance on objects with internal degrees of freedom. PLoS Comput Biol 5(6):e1000419

15. Bays PM, Wolpert DM (2007) Computational principles of sensorimotor control that minimize uncertainty and variability. J Physiol 578(Pt 2):387-396

16. Flash T, Hochner B (2005) Motor primitives in vertebrates and invertebrates. Curr Opin Neurobiol 15(6):660-666

17. Mussa-Ivaldi F, Solla S (2004) Neural primitives for motion control. IEEE J Ocean Eng 29(3):640-650

18. Harris CM (2009) Biomimetics of human movement: functional or aesthetic? Bioinspiration Biomimetics 4(3):033001

19. Viviani P, Flash T (1995) Minimum-jerk, two-thirds power law, and isochrony: converging approaches to movement planning. J Exp Psychol Hum Percept Perform 21(1):32-53
20. Flash T, Meirovitch Y, Barliya A (2013) Models of human movement: trajectory planning and inverse kinematics studies. Robot Auton Syst 61(4):330-339

21. Harris C (1998) Signal-dependent noise determines motor planning. Nature 394:780-784

22. Grush R (2004) The emulation theory of representation: motor control, imagery, and perception. Behav Brain Sci 27(3):377-396, discussion 396-442

23. Wolpert DM, Miall RC, Kawato M (1998) Internal models in the cerebellum. Trends Cogn Sci 2(9):338-347

24. Macadam CC (2003) Understanding and modeling the human driver. Veh Syst Dyn 40(1-3):101-134

25. Plöchl M, Edelmann J (2007) Driver models in automobile dynamics application. 45:(7-8):699-741

26. Bertolazzi E, Biral F, Da Lio M, Saroldi A, Tango F (2010) Supporting drivers in keeping safe speed and safe distance: the SASPENCE subproject within the European framework programme 6 integrating project PReVENT. IEEE Trans Intell Transp Syst 11(3): $525-538$

27. Cossalter V, Da Lio M, Biral F, Fabbri L (1998) SAE TECHNICAL evaluation of motorcycle maneuverability with the optimal maneuver method. Eng Conf 724

28. Biral F, Da Lio M, Bertolazzi E (2005) Combining safety margins and user preferences into a driving criterion for optimal control-based computation of reference maneuvers for an ADAS of the next generation. In: IEEE Intelligent Vehicles Symposium, Proceedings, vol. 2005 , pp. 36-41

29. Biral F, da Lio M, Lot R, Sartori R (2010) An intelligent curve warning system for powered two wheel vehicles. Eur Transp Res Rev 2(3):147-156

30. Cossalter V, Da Lio M, Lot R, Fabbri L (1999) A general method for the evaluation of vehicle manoeuvrability with special emphasis on motorcycles. Veh Syst Dyn 31(2):113-135

31. Ritchie ML, McCoy WK, Welde WL (1968) A study of the relation between forward velocity and lateral acceleration in curves during normal driving. Hum Factors 10(3):255-258

32. Van Winsum W, Godthelp H (1996) Speed choice and steering behaviour in curve driving. Hum Factors 38(3):434-441

33. Emmanuel F, Navin F (1998) Automobiles on horizontal curves. Transp Res Rec 98:50-56

34. Levison WH (1998) Interactive highway safety design model: issues related to driver modeling. Transp Res Rec 1631:20-27

35. Savkoor AR, Ausejo S (2000) Analysis of driver's steering and speed control strategies in curve negotiation. Veh Syst Dyn 33(SUPPL):94 109

36. Reymond G, Kemeny A, Droulez J, Berthoz A (2001) Role of lateral acceleration in curve driving: driver model and experiments on a real vehicle and a driving simulator. Hum Factors J Hum Factors Ergon Soc 43(3):483-495

37. Levinson W (2007) "Development of a driver vehicle module for the interactive highway safety design model,"[Online]. Available: http:// www.fhwa.dot.gov/publications/research/safety/08019/08019.pdf. [Accessed: 31-May-2013]

38. Cafiso S, Cerni G (2012) New approach to defining continuous speed profile models for two-lane rural roads. Transp Res Rec J Transp Res Board 2309(1):157-167

39. Fancher P, Bareket Z, Ervin R (2001) Human-centered design of an ACC-with-braking and forward-crash-warning system. Veh Syst Dyn 36(2-3):203-223

40. Moon S, Yi K (2008) Human driving data-based design of a vehicle adaptive cruise control algorithm. Veh Syst Dyn 46(8):661-690

41. Rice RS (1973) Measuring car-driver interaction with the g-g diagram. SAE Technical Papers 
42. Hisaoka Y, Yamamoto M, Okada A (1999) Closed-loop analysis of vehicle behavior during braking in a turn. JSAE Rev 20:537-542

43. Bartlett W, Masory O, Wright B (2000) Driver abilities in closed course testing. SAE Technical Papers

44. Hollnagel E (2002) Cognition as control: a pragmatic approach to the modelling of joint cognitive systems. Control 9:1-23

45. Hollnagel E, Woods DD (2005) Joint cognitive systems. Taylor \& Francis Group, LLC, pp. 113-133

46. Michon JA (1985) A critical view of driver behavior models: what do we know, what should we do? Hum Behav Traffic Saf 485-520
47. “Test Track (Google Maps).” [Online]. Available: http://goo.gl/maps/ mzfBs

48. Levison WH, Bittner AC Jr, Campbell JL, Schreiner C (2002) Modification and partial validation of the driver/vehicle module. Transp Res Rec 1803:52-58

49. Hicheur H, Vieilledent S, Richardson MJE, Flash T, Berthoz A (2005) Velocity and curvature in human locomotion along complex curved paths: a comparison with hand movements. Exp Brain Res Experimentelle Hirnforschung Expérimentation Cérébrale 162(2): $145-154$ 